\title{
Synthesis, spectroscopic characterization and in vitro antitumor activity of new trans 1-heteroaryl-2-(1-methylpyridinium-2-yl) ethylenes
}

\author{
Francesco P. Ballistreri ${ }^{\mathrm{a}}$, Vincenza Barresi ${ }^{\mathrm{a}}$, Giuseppe Consiglio ${ }^{\mathrm{b}}$, Cosimo G. Fortuna ${ }^{\mathrm{a}}$, \\ Maria L. Longo ${ }^{a}$, Giuseppe Musumarra ${ }^{* a}$ \\ ${ }^{a}$ Dipartimento di Scienze Chimiche, Università di Catania, Viale A.Doria,6 95125 Catania, Italy \\ ${ }^{b}$ Dipartimento di Metodologie Fisiche e Chimiche per l'Ingegneria, Viale A.Doria,6 95125 \\ Catania, Italy \\ E-mail: gmusumarra@dipchi.unict.it
}

(received 20 Dec 03; accepted 20 Jun 03; published on the web 22 Jun 03)

\begin{abstract}
The synthesis and spectroscopic characterization of trans 1-heteroaryl-2-(1-methylpyridinium-2yl) ethylene iodides is reported. The 5-(4-bromophenyl)furan derivative exhibited the highest in vitro antitumor activity against MCF7 (breast) and LNCap (prostate) carcinoma cells. Solvatochromic and NMR shifts provided evidence for modulation of electronic parameters which could be used to optimize frequency emission and conversion efficiency in such push-pull conjugated systems, which are potential components of non linear optics materials.
\end{abstract}

Keywords: Aromatic heterocycles, bio-organic, cytotoxicity, pharmaceuticals, pyridine

\section{Introduction}

We have recently reported the synthesis of 2,6-di-[2-(heteroaryl)vinyl]pyridines ${ }^{1}$ and pyridiniums, ${ }^{2}$ where three heteroaromatics rings are linked by two ethylenic double bonds exerting a "spacing" function. Antitumor in vitro tests against the 60 National Cancer Institute (NCI) tumor cell lines panel pointed out antiproliferative effects particularly evident for MCF7 mammary adenocarcinoma cells and multivariate analysis of DNA microarray data for responsive tumor cell lines suggests a mechanistic pathway involving polyamine biosynthesis and prolactin signal transduction. ${ }^{3}$

We here report the synthesis and spectroscopic characterization of water soluble trans 1heteroaryl-2-(1-methylpyridinium-2-yl) ethylenes 1-12 as well as in vitro antitumour tests against selected cell lines. 


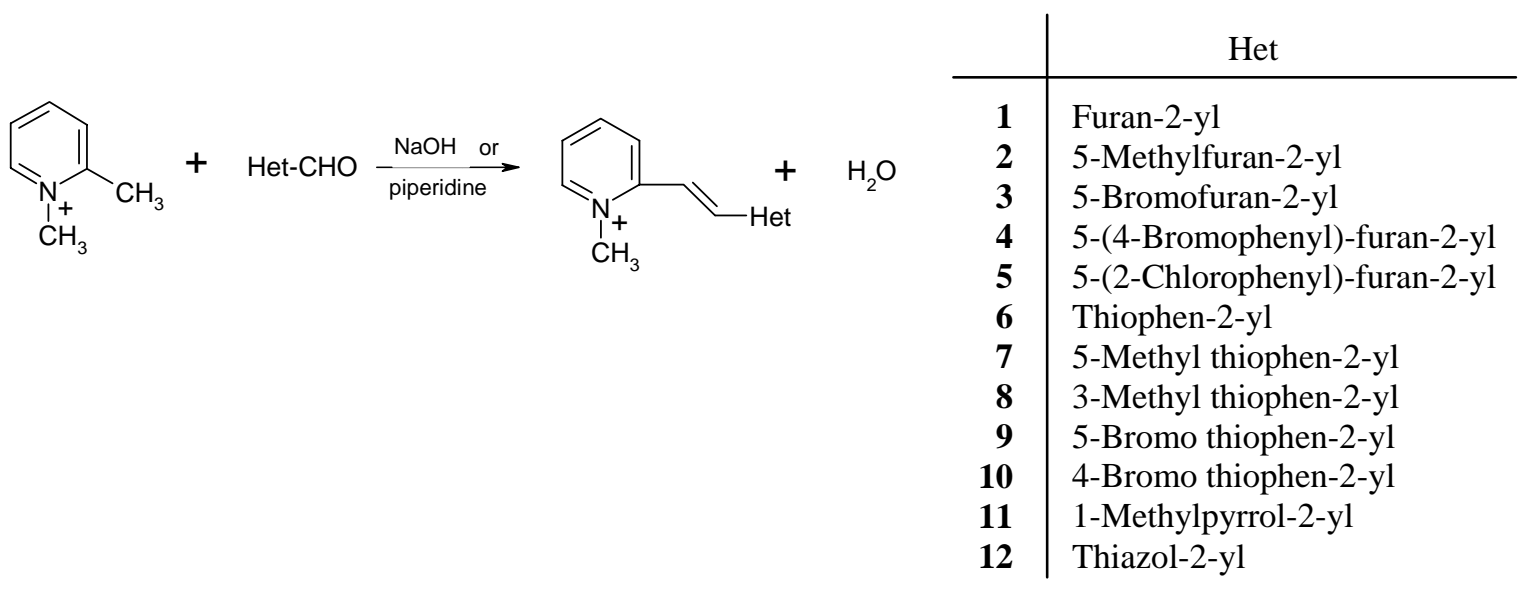

Compounds 1 - 12, possessing heteroaromatic moieties with different electron donating abilities linked by a vinyl to the strong electron withdrawing pyridinium ring, belong to the class of the so called push-pull (donor-acceptor, D-A) molecules. Such push-pull conjugated systems are possible candidate components of non linear optics, which are already well documented for push-pull ethenes for example 4-dimethylamino-4'-nitrostilbene (DANS) ${ }^{4}$ and for heterocyclic analogues with $\pi$-excessive and $\pi$-deficient heteroaromatics (D-A systems). ${ }^{5}$ It was recently shown ${ }^{6}$ that methyl substituted 1-methylpyrroles $\mathbf{1 1}$ are able to efficiently convert the emission of a cheap IR laser into that of a valuable visible laser. In this context we here also report solvatochromic and NMR shifts providing evidence for a wide modulation of electronic parameters which could be used to optimize frequency emission and conversion efficiency.

\section{Results and Discussion}

The synthesis of trans 1-heteroaryl-2-(1-methylpyridinium-2-yl) ethylenes 1-12 is straightforward and can be easily achieved by condensation of (1,2,-dimethyl)pyridinium iodide with heteroaromatic aldehydes (see Experimental section). As expected, the pyridinium $\alpha$ methyls are quite reactive towards base due to the strong electron withdrawing effect of the positively charged ring nitrogen.

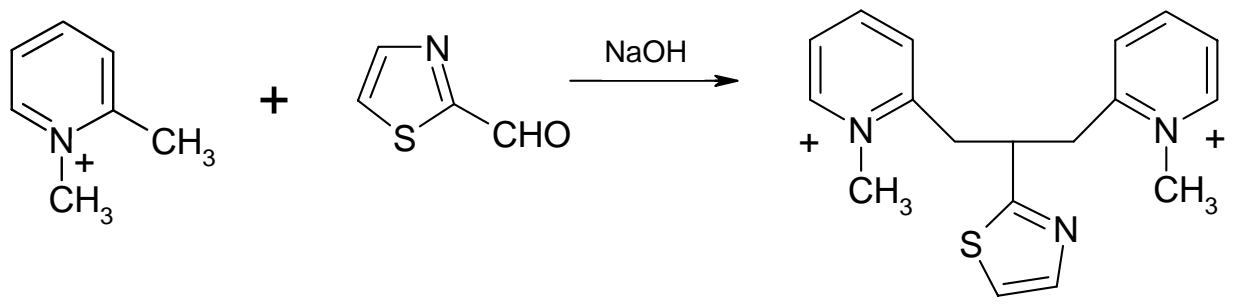


Under appropriate experimental conditions, outlined in the experimental section, pure trans isomers 1 - 11 were obtained, as evidenced by the ethylenic protons $\mathrm{J}$ coupling constants in the NMR spectra (see Experimental). Reaction of 2-thiazolaldehyde with 1-methylpicolinium iodide in the presence of one drop 5\% $\mathrm{NaOH}$ afforded a mixture of the expected ethylene $\mathbf{1 2}$ and of bis cation 13 (1:5 ratio) as determined by NMR signals. On increasing the $\mathrm{NaOH}$ concentration up to $10 \%$, pure bis cation 13 was obtained in $49 \%$ yield.

Compounds 1-11 possess electron donating (furan, thiophene and pyrrol) moieties linked by a vinyl linker to the pyridinium ring, while the thiazole derivative $\mathbf{1 2}$ exerts a weak electronwithdrawing effect. Accordingly, all $\mathrm{H}_{\mathrm{a}}$ ethylenic NMR shifts resonate at lower fields with respect to $\mathrm{H}_{\mathrm{b}}$, and, among them, that of $\mathbf{1 2}(8.13 \mathrm{ppm})$ is the most downfield shifted (see Experimental).

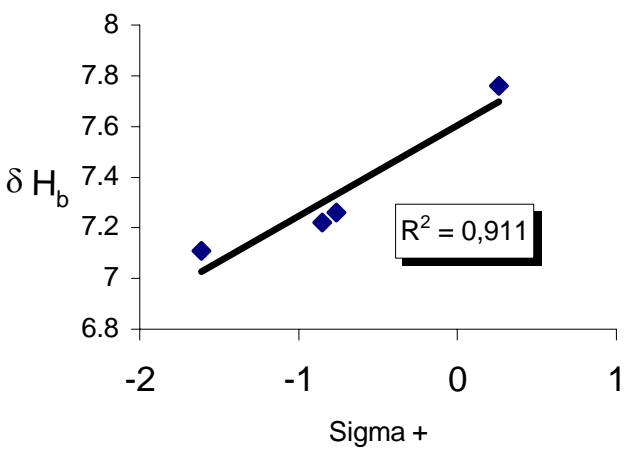

Figure 1. Correlation between tra $\delta \mathrm{H}_{\mathrm{b}}$ and $\sigma^{+}$constants from solvolysis reactions (-0.85 for furan-2-yl, -0.76 for thiophen-2-yl, -1.61 for pyrrol-2-yl and 0.26 for thiazol-2-yl).

Figure 1 shows a good correlation $\left(\mathrm{R}^{2}=0.911\right)$, in a wide range of $\sigma^{+}\left(\sigma^{+}\right.$constants derived from solvolysis reactions. ${ }^{7,8}$ ), between $\mathrm{H}_{\mathrm{b}}$ ethylenic proton shifts for unsubstituted derivatives $\mathbf{1}$, 6, 11 and 12. Derivatives with extended conjugation are expected to exhibit significant solvatochromic shifts. The absorption maxima of cations $\mathbf{1}$ - $\mathbf{1 1}$ in protic and aprotic solvent of various polarity are reported in Table 1.

Water-chloroform solvatochromic shifts above $20 \mathrm{~nm}$ are observed for cations 2, 5, 7, and 11, in agreement with the high donating ability of the five membered moieties in such push-pull systems. Solvatochromic shifts available for unsubstituted derivatives 1, 6 and $\mathbf{1 1}$ follow closely the order of $\sigma^{+}$constants for furan, thiophene and pyrrole moieties derived from solvolysis reactions, ${ }^{7,8}$ although the low number of points and their distribution suggest great caution in using the term correlation $\left(\mathrm{R}^{2}=0.963\right)$.

Solvatochromic and NMR shifts reported in the present paper span a wide range of variation (up to $1.87 \sigma^{+}$units) in the electronic effects of such push-pull conjugated molecules, providing the possibility to widely modulate electronic parameters, known to be essential in optimizing and 
tuning frequency emission and conversion efficiency. ${ }^{6}$ The above finding indicates pointing out cations 1-12 as new possible candidate components for non linear optics.

Another purpose of the present work is to test the in vitro antitumor activity of the title trans 1-heteroaryl-2-(1-methylpyridinium-2-yl)ethylenes and to compare the results with those of previous studies on the activity of 2,6-di-[2-(heteroaryl)vinyl]pyridines ${ }^{9}$ and pyridiniums ${ }^{3}$ where three heteroaromatics rings are linked by two ethylenic double bonds.

Table 1. Absorption maxima for 1-heteroaryl-2-(1-methylpyridinium-2-yl) ethylenes 1-11 ${ }^{\mathrm{a}}$ in different solvents

\begin{tabular}{|c|c|c|c|c|}
\hline Compounds \ Solvents & $\mathrm{CHCl}_{3}$ & $\mathrm{EtOH}$ & $\mathrm{MeOH}$ & $\mathrm{H}_{2} \mathrm{O}$ \\
\hline $\mathbf{1}$ & 399.08 & 382.27 & 381.95 & 381.06 \\
& $(3.94)$ & $(3.79)$ & $(3.79)$ & $(3.91)$ \\
\hline $\mathbf{2}$ & 402.34 & 384.79 & 384.53 & 382.03 \\
& $(3.95)$ & $(3.69)$ & $(3.81)$ & $(3.87)$ \\
\hline $\mathbf{3}$ & 393.91 & 377.92 & 378.59 & 375.14 \\
& $(3.74)$ & $(3.81)$ & $(3.83)$ & $(3.83)$ \\
\hline $\mathbf{4}$ & 438.33 & 425.59 & 420.80 & 420.42 \\
& $(4.32)$ & $(4.23)$ & $(4.14)$ & $(4.25)$ \\
\hline $\mathbf{5}$ & 428.72 & - & 410.85 & 403.59 \\
& $(4.41)$ & & $(4.42)$ & $(4.36)$ \\
\hline $\mathbf{6}$ & 380.70 & 374.11 & 372.12 & 366.24 \\
& $(4.09)$ & $(4.11)$ & $(4.14)$ & $(4.18)$ \\
\hline $\mathbf{7}$ & 387.82 & 386.84 & 381.21 & 363.94 \\
& $(4.01)$ & $(4.11)$ & $(4.10)$ & $(4.06)$ \\
\hline $\mathbf{8}$ & 387.27 & 371.62 & 374.645 & 371.81 \\
& $(4.02)$ & $(3.85)$ & $(3.70)$ & $(3.94)$ \\
\hline $\mathbf{9}$ & 376.90 & 365.45 & 372.76 & 364.90 \\
& $(3.81)$ & $(3.83)$ & $(3.74)$ & $(3.85)$ \\
\hline $\mathbf{1 0}$ & 387.89 & 374.26 & 374.00 & 372.09 \\
& $(4.36)$ & $(4.33)$ & $(4.37)$ & $(4.33)$ \\
\hline $\mathbf{1 1}$ & 438.33 & 428.23 & 424.87 & 411.72 \\
& $(4.19)$ & $(4.18)$ & $(4.16)$ & $(4.16)$ \\
\hline
\end{tabular}

${ }^{\mathrm{a}} \log \varepsilon$ in parentheses. 


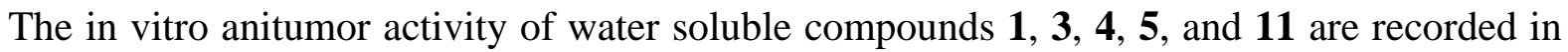
Table 2.

Table 2. In vitro antitumor activities, expressed as $\log \mathrm{GI}_{50}$, for MCF7 and LNCap cell lines

\begin{tabular}{|l|l|l|l|l|l|l|}
\hline Cell line/Compounds & $\mathbf{1}$ & $\mathbf{3}$ & $\mathbf{4}$ & $\mathbf{5}$ & $\mathbf{1 1}$ & $\mathbf{P F}_{\mathbf{2}}$ \\
\hline MCF7 (breast) & -5.00 & -5.10 & -7.75 & -5.65 & -4.82 & -7.23 \\
\hline LNCap (prostate) & -4.00 & -4.50 & -6.00 & -5.00 & -4.00 & -5.38 \\
\hline
\end{tabular}
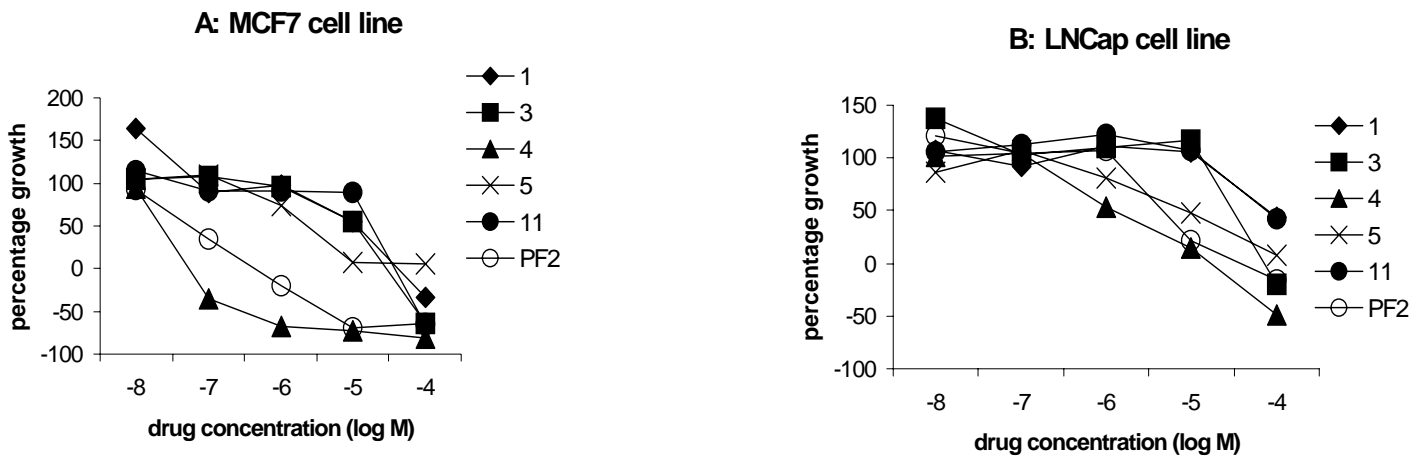

Figure 2. Dose - response curves of the antiproliferative activities of compounds $\mathbf{1}(\bullet), \mathbf{3}(\bullet), \mathbf{4}(\bullet), \mathbf{5}(\times), \mathbf{1 1}(\bullet)$ and $\mathrm{PF}_{2}(\mathrm{o})$ on MCF7 (A) and LNCap (B) cell lines.

The anti-proliferative activity of typical derivatives was tested against two tumor cell lines, breast carcinoma (MCF7) and prostate carcinoma (LNCap). The percent of growth and the inhibition exerted by different doses $(0.01-100 \mu \mathrm{M})$ are shown in figure 2 and $\log \mathrm{GI}_{50}$ values, calculated as described in the experimental section are recorded in Table 2. All compounds in Table 2 are more active towards MCF-7 than LNCap cell lines. Data relative to 2,6-di-[2-(furan2-yl)vinyl]pyridinium iodide $\left(\mathrm{PF}_{2}\right)$, which was the most active in previous in vitro tests, ${ }^{3}$ are also reported for comparisons. Towards MCF-7 and LNCap carcinoma cells (Table 2) the 1-[5-(4Bromophenyl)-furan-2-yl]-2-(1-methyl-pyridinium-2-yl) ethylene (ligand 4) exhibits highest activity but slightly better than the $\mathrm{PF}_{2}$. Figure 2 shows that, in addition to the antiproliferative effects ( $\log \mathrm{GI}_{50}$ ), the bromophenyl derivative 4 exhibits a significant cytotoxic activity. In fact only $25 \%$ MCF7 carcinoma cells survive at $10^{-6} \mathrm{M}$ concentration. This finding points to 4 as a possible lead compound for future antitumor studies. In conclusion, 1-heteroaryl-2-(1methylpyridinium-2-yl)ethylenes 1-11 reported in the present work exhibit interesting potential applications both as candidates for more detailed in vivo antitumor studies and as components for non linear optics due to a wide modulation of the donor heteroaromatic moiety capabilities. 


\section{Experimental Section}

General Procedures. Heteroaromatic carboxaldehydes, Aldrich commercial products, were used without further purification.UV-Vis spectra were recorded on a Perkin Elmer Lambda 2S spectrometer . ${ }^{1} \mathrm{H}$ NMR spectra were recorded on a Varian Unity Inova spectrometer operating at $500 \mathrm{MHz}$, at $25^{\circ} \mathrm{C}$ in (CD3)2SO using TMS as internal standards. The spectral width was set to $5,000 \mathrm{~Hz}$, with an excitation pulse of 60 degrees, an acquisition time of $3.5 \mathrm{~s}$ and a digital resolution after zero-filling of $0.15 \mathrm{~Hz} / \mathrm{pt}$. Fast Atom Bombardment (FAB) mass spectra were recorded on a double focusing Kratos MS 50 mass spectrometer equipped with the standard FAB source and the Maspec2 data acquisition and processing system (Mass Spectrometry Services Ltd). Mass resolution was 3.000 and the matrix 3-nitro-benzyl alcohol. Mass spectra ESI were recorded on a LCQ Deca Finnigan system with a flow of $5 \mu \mathrm{l} / \mathrm{min}$.Compounds 1-13, all iodide salts, were obtained by reacting in equimolar amounts 1,2 dimethylpyridinium iodide with the appropriate heteroaromatic aldehyde in the presence of a base. Details on the synthetic conditions (base, solvent, reaction time and temperature) and products characterization are reported below:

1-(Furan-2-yl)-2-(1-methylpyridinium-2-yl) ethylene iodide (1). Piperidine, EtOH, 8 hours, $25^{\circ} \mathrm{C}$. Yield 23\%; (Green prisms from ethanol); mp 218-219 ${ }^{\circ} \mathrm{C}$ (lit., ${ }^{10} 204-206^{\circ} \mathrm{C}$, no NMR available); ${ }^{1} \mathrm{H}$ NMR , (DMSO-d ${ }_{6}$ ): $\delta=7.86$ (d, $J=15.5 \mathrm{~Hz}, 1 \mathrm{H}$, a-H), 7,22 (d, $J=16 \mathrm{~Hz}, 1 \mathrm{H}, \mathrm{b}-\mathrm{H}$ ), 4.32 (s, 3H, N ${ }^{+} \mathrm{Me}$ ), 8.47 (m, 1H, 3-H), 8.45 (m, 1H, 4-H), 7.86 (m, 1H, 5-H), 8.88 (d, J=6.5 Hz, 1H, 6-H), 7.05 (d, $J=3.5 \mathrm{~Hz}, 1 \mathrm{H}, 3$ '-H), 6.73 (dd, $J=2 \mathrm{~Hz}, 1 \mathrm{H}, 4^{\prime}-\mathrm{H}$ ), 7.97 (d, $J=1.5 \mathrm{~Hz}, 1 \mathrm{H}, 5$ 'H). MS: positive FAB: $\left(\mathrm{M}^{+}=185.9\right)$; Anal. Calcd for $\mathrm{C}_{12} \mathrm{H}_{12} \mathrm{INO}$ : C, 46.03; H, 3.86; N, 4.47. Found: C, 46.15; H, 3.78; N, 4.50.

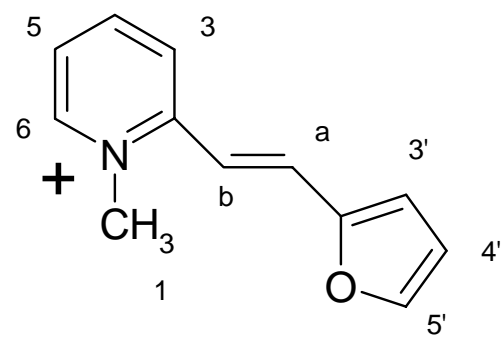

1-(5-Methylfuran-2-yl) 2-(1-methylpyridinium-2-yl) ethylene iodide (2). 5\% $\mathrm{NaOH}, \mathrm{EtOH}, 5$ min, reflux. Yield 54\%; (Yellow prisms from ethanol); mp 260-261 ${ }^{\circ} \mathrm{C}$; ${ }^{1} \mathrm{H}$ NMR (DMSO- $\mathrm{d}_{6}$ ), $\delta=7.78(\mathrm{~d}, J=16 \mathrm{~Hz}, 1 \mathrm{H}, \mathrm{a}-\mathrm{H}), 7,06$ (d, $J=15.5 \mathrm{~Hz}, 1 \mathrm{H}, \mathrm{b}-\mathrm{H}), 4.30$ (s, 3H, N $\left.{ }^{+} \mathrm{Me}\right), 8.43$ (m, 1H, 3-H), 8.41 (m, 1H, 4-H), 7.82 (m, 1H, 5-H), 8.85 (d, J=6.5 Hz, 1H, 6-H), 6.96 (d, J=3.5 Hz, 1H, 3’-H), 6.61 (d, J=3 Hz, 1H, 4'-H), 2.40 (s, 3H, 5'-Me). MS: ESI (M+ = 200); Anal. Calcd for $\mathrm{C}_{13} \mathrm{H}_{14} \mathrm{INO}$ : C, 38.36; H, 3.47; N, 3.44. Found: C, 37.40; H, 3.51; N, 3.50. 


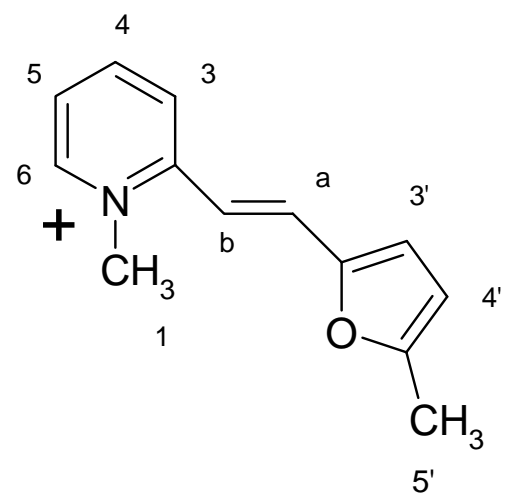

1-(5-Bromofuran-2-yl)-2-(1-methylpiridinium-2-yl) ethylene iodide (3). 20\% $\mathrm{NaOH}, \mathrm{EtOH}, 2$ hours, reflux. Yield 49\%; (Yellow needles from ethanol); mp $>200{ }^{\circ} \mathrm{C}$; ${ }^{1} \mathrm{H}$ NMR (DMSO- $\mathrm{d}_{6}$ ): $\delta=7.78$ (d, $J=16 \mathrm{~Hz}, 1 \mathrm{H}, \mathrm{a}-\mathrm{H}), 7.21$ (d, $J=16 \mathrm{~Hz}, 1 \mathrm{H}, \mathrm{b}-\mathrm{H})$, 4.34 (s, 3H, N ${ }^{+} \mathrm{Me}$ ), 8.91 (d, $J=6$ Hz,1H, 6-H), 8.48 (t, J=8 Hz, 1H, 4-H), 8.44 (d, $J=8.5$ Hz, 1H, 3-H),7.89 (m, J=6.25 Hz, 1H, 5H),7.09 (d, J=4 Hz, 1H, 3'-H), 6.87 (d, J=3.5 Hz, 1H, 4'-H). MS: positive FAB (M+ Br $79=264$, $\mathrm{M}+\mathrm{Br}_{81}=266$ ); ); Anal. Calcd for $\mathrm{C}_{12} \mathrm{H}_{11}$ BrINO: C, 36.77; H, 3.86; N, 3.57. Found: C, 36.82; $\mathrm{H}$, 3.74; N, 3.63 .

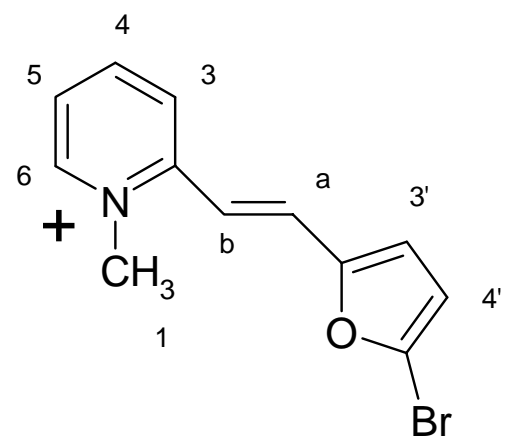

1-[5-(4-Bromophenyl)-furan-2-yl]-2 -(1-methyl-pyridinium-2-yl) ethylene iodide (4). 10\% $\mathrm{NaOH}, \mathrm{EtOH}, 10 \mathrm{~min}$, reflux. Yield 42\%; (Orange needles from aqueous-ethanol); mp 260$263{ }^{\circ} \mathrm{C} ;{ }^{1} \mathrm{H}$ NMR (DMSO-d $)$ ) $\delta=7.88$ (d, $\left.J=16 \mathrm{~Hz}, 1 \mathrm{H}, \mathrm{a}-\mathrm{H}\right), 7.37$ (d, $\left.J=16 \mathrm{~Hz}, 1 \mathrm{H}, \mathrm{b}-\mathrm{H}\right), 4.37$ (s, 3H, N ${ }^{+} \mathrm{Me}$ ), 8.48 (d, 1H, 3-H), 8.46 (dd, $\left.J=8.5 \mathrm{~Hz}, 1 \mathrm{H}, 4-\mathrm{H}\right), 7.87$ (m, 1H, 5-H), 8.87 (d, J=6.5 Hz, 1H, 6-H), 7.31 (d, J=4 Hz, 1H, 3'-H), 7.16 (d, J=4 Hz, 1H, 4'-H), 7.07 (m, 2H, 3' '-H, 5' '-H), 7.70 (d, J=8.5 Hz, 2H, 2'’-H, 6'’-H). MS: positive FAB ( $\mathrm{M}^{+} \mathrm{Br}_{79}=339.7 ; \mathrm{M}^{+} \mathrm{Br}_{81}=341.7$ ). Anal. Calcd for $\mathrm{C}_{18} \mathrm{H}_{15}$ BrINO: C, 46.18; H, 3.23; N, 3.00. Found: C, 46.24; H, 3.19; N, 3.12. 


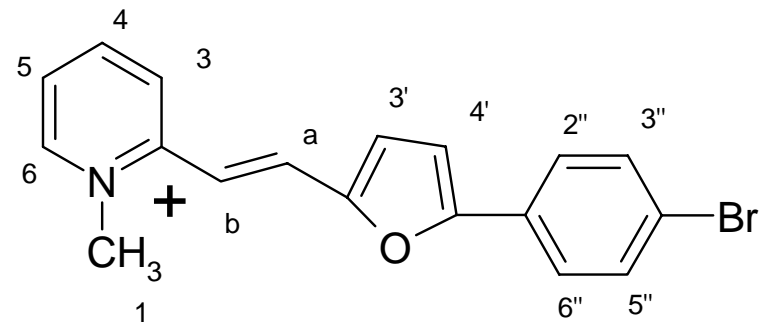

1-[5-(2-Chlorophenyl)- furan- 2 -yl]-2-(1-methyl-pyridinium-2-yl) ethylene iodide (5). 20\% $\mathrm{NaOH}, \mathrm{EtOH}, 10 \mathrm{~min}$, reflux. Yield 34\%; (Orange needles from ethanol); mp 190-193 ${ }^{\circ} \mathrm{C} ;{ }^{1} \mathrm{H}$ NMR (DMSO-d ${ }_{6}$ ), $\delta=7.90$ (d, $\left.J=15.5 \mathrm{~Hz}, 1 \mathrm{H}, \mathrm{a}-\mathrm{H}\right), 7.40$ (d, $\left.J=15.5 \mathrm{~Hz}, 1 \mathrm{H}, \mathrm{b}-\mathrm{H}\right), 4.38$ (s, $3 \mathrm{H}$, $\mathrm{N}^{+} \mathrm{Me}$ ), 8.48 (d, 1H, 3-H), 8.50 (dd, J=7.5 Hz, 1H, 4-H), 7.88 (m, 1H, 5-H), 8.90 (d, J=6 Hz, 1H, 6-H), 6.17 (d, $J=3.5 \mathrm{~Hz}, 1 \mathrm{H}, 3$ '-H), 6.34 (d, $J=4 \mathrm{~Hz}, 1 \mathrm{H}, 4$ '-H), 7.07 (dd, $J=8 \mathrm{~Hz}, 1 \mathrm{H}, 3$ ''-H), 6.39 (m, $J=8 \mathrm{~Hz}, 1 \mathrm{H}, 4$ '’-H), 6.47 (m, $J=8 \mathrm{~Hz}, 1 \mathrm{H}, 5$ '”-H), 6.58 (dd, $J=8 \mathrm{~Hz}, 1 \mathrm{H}, 6$ ''-H). MS: positive $\mathrm{FAB}\left(\mathrm{M}^{+} \mathrm{Cl}_{35}=296.1 ; \mathrm{M}^{+} \mathrm{Cl}_{37}=298.1\right)$. Anal. Calcd for $\mathrm{C}_{18} \mathrm{H}_{15} \mathrm{ClINO} \mathrm{C}, 51.03 ; \mathrm{H}$, 3.57; N, 3.30. Found: C, 50.09; H, 3.62; N, 3.38 .

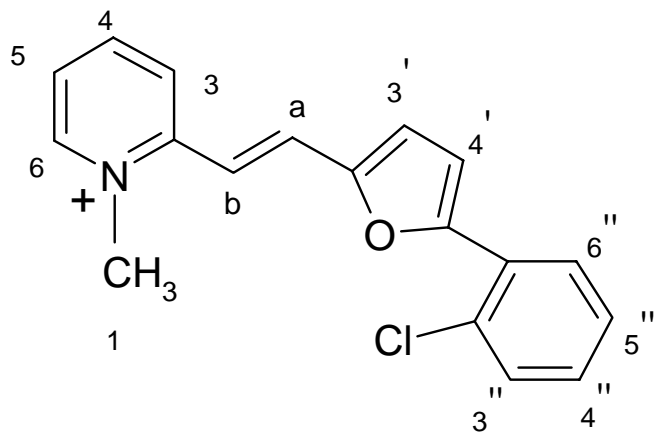

1-(Thiophen-2-yl)-2-(1-methylpyridinium-2-yl) ethylene iodide (6). $20 \% \mathrm{NaOH}$, EtOH, 1 hour, reflux. Yield 63\%; (Brown needles from ethanol); mp 225-229 $\mathrm{C}$ (lit., ${ }^{11} 231-232^{\circ} \mathrm{C}$, no NMR available); ${ }^{1} \mathrm{H}$ NMR, $\left(\right.$ DMSO-d $\left._{6}\right) \delta=8.16$ (d, $\left.J=15.5 \mathrm{~Hz}, 1 \mathrm{H}, \mathrm{a}-\mathrm{H}\right), 7.26$ (d, $J=15.5 \mathrm{~Hz}$, $1 \mathrm{H}, \mathrm{b}-\mathrm{H}$ ) , 4.34 (s, 3H, N ${ }^{+} \mathrm{Me}$ ), 8.88 (d, J=6.5 Hz, 1H, 6-H), 7.24 (dd, 1H, 3-H), 8.47 (dd, 1H, 4H), 6.70 (d, 1H, 5-H), 7.85-7.88 (m, 3H, 3'-H, 4'-H, 5'-H). MS: positive FAB $\left(\mathrm{M}^{+}=201.6\right)$. Anal. Calcd for $\mathrm{C}_{12} \mathrm{H}_{12} \mathrm{INS}$ : C, 43.78; H, 3.67; N, 4.25; S, 9.74. Found: C, 43.60; H, 3.74; N, 4.34; S, 9.70 . 


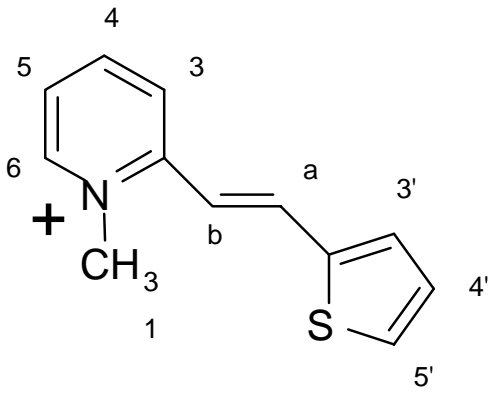

1-(5-Methylthiophen-2-yl)-2-(1-methylpyridinium-2-yl) ethylene iodide (7). $5 \% \mathrm{NaOH}$, EtOH, 10 min, reflux. Yield 11\%; (Brown prisms from ethanol); mp 271-273 ${ }^{\circ} \mathrm{C} ;{ }^{1} \mathrm{H}$ NMR (DMSO-d d $_{6}, \delta=8.09$ (d, $J=16 \mathrm{~Hz}, 1 \mathrm{H}, \mathrm{a}-\mathrm{H}$ ), 7.16 (d, $J=16 \mathrm{~Hz}, 1 \mathrm{H}, \mathrm{b}-\mathrm{H}$ ), 4.30 (s, 3H, N ${ }^{+} \mathrm{Me}$ ), 8.45 (d, $J=8 \mathrm{~Hz}, 1 \mathrm{H}, 3-\mathrm{H}$ ), 8.42 (t, $J=7,5 \mathrm{~Hz}, 1 \mathrm{H}, 4-\mathrm{H}), 7.82$ (t, $J=6.5 \mathrm{~Hz}, 1 \mathrm{H}, 5-\mathrm{H}), 8.85$ (d, $J=6.5$ Hz, 1H, 6-H), 7.46 (d, $J=4 \mathrm{~Hz}, 1 \mathrm{H}, 3$ '-H), 6.94 (d, $J=3 \mathrm{~Hz}, 1 \mathrm{H}, 4^{\prime}-\mathrm{H}$ ). 2.52 (s, 3H, 5'-Me). MS: ESI $\left(\mathrm{M}^{+}=216\right)$. Anal. Calcd for $\mathrm{C}_{13} \mathrm{H}_{16} \mathrm{INS}$ : C, 45.23; H, 4.67; N, 4.06; S, 9.29. Found: $\mathrm{C}$, 45.32; H, 4.00; N, 4.12; S, 9.20.

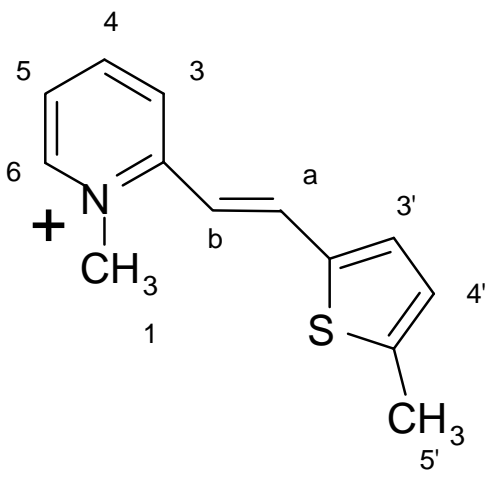

1-(3-Methylthiophen-2-yl)-2-(1-methylpyridinium-2-yl) ethylene iodide (8). $10 \% \mathrm{NaOH}$, $\mathrm{EtOH}, 10 \mathrm{~min}$, reflux. Yield 45\%; (Yellow needles from ethanol); mp 259-262 ${ }^{\circ} \mathrm{C} ;{ }^{1} \mathrm{H}$ NMR (DMSO-d ${ }_{6}$ ) , $\delta=8.08$ (d, $\left.J=15.5 \mathrm{~Hz}, 1 \mathrm{H}, \mathrm{a}-\mathrm{H}\right), 7.04$ (d, $\left.J=15.5 \mathrm{~Hz}, 1 \mathrm{H}, \mathrm{b}-\mathrm{H}\right), 4.31$ (s, 3H, N ${ }^{+} \mathrm{Me}$ ), 8.61 (d, $J=8 \mathrm{~Hz}, 1 \mathrm{H}, 3-\mathrm{H}$ ), 8.45 (t, $J=8 \mathrm{~Hz}, 1 \mathrm{H}, 4-\mathrm{H}$ ), 7.85 (t, $J=6.5 \mathrm{~Hz}, 1 \mathrm{H}, 5-\mathrm{H}), 8.85$ (d, $J=6$ Hz, 1H, 6-H), 2.48 (s, 3H, 3'-Me), 7.06 (d, J=5 Hz,1H, 4'-H), 7.73 (d, J=4.5 Hz, 1H, 5'-H). MS: ESI $\left(\mathrm{M}^{+}=216\right.$ ). Anal. Calcd for $\mathrm{C}_{13} \mathrm{H}_{16} \mathrm{INS}$ : C, 45.23; H, 4.67; N, 4.06; S, 9.29. Found: $\mathrm{C}$, 45.41; H, 4.56; N, 4.12; S, 9.10. 


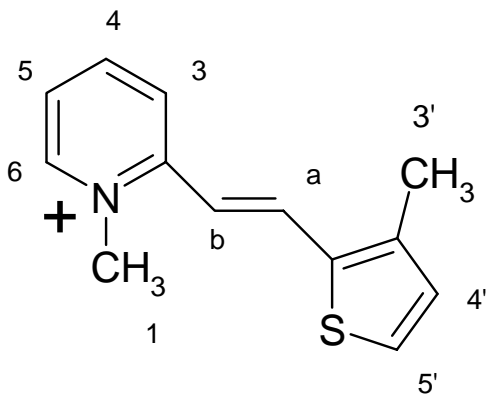

1-(5 Bromothiophen-2-yl)-2-(1-methylpyridinium-2-yl) ethylene iodide (9). $10 \% \mathrm{NaOH}$, EtOH, 5 min, reflux. Yield 73\%; (Yellow prisms from ethanol); mp 241-242 ${ }^{\circ} \mathrm{C}$; ${ }^{1} \mathrm{H}$ NMR (DMSO-d $)_{6}$ ), $\delta=8.06$ (d, J=16 Hz, 1H, a-H), 7.22 (d, J=16 Hz, 1H, b-H), 4.31 (s, 3H, N Me), 8.43 (m, 2H, 3-H, 4-H), 7.87 (t, $J=6.5$ Hz, 1H, 5-H), 8.85 (d, $J=6$ Hz, 1H, 6-H), 7.37 (d, $J=4$ Hz, $1 \mathrm{H}, 3$ '-H), 7.47 (d, J=4 Hz, 1H 4'-H). MS: ESI ( $\mathrm{M}^{+}=281$ ). Anal. Calcd for $\mathrm{C}_{12} \mathrm{H}_{11} \mathrm{BrINS}$ : C, 35.32; H, 2.72; N, 3.43; S, 7.85. Found: C, 35.41; H, 2.65; N, 3.56; S, 7.81.

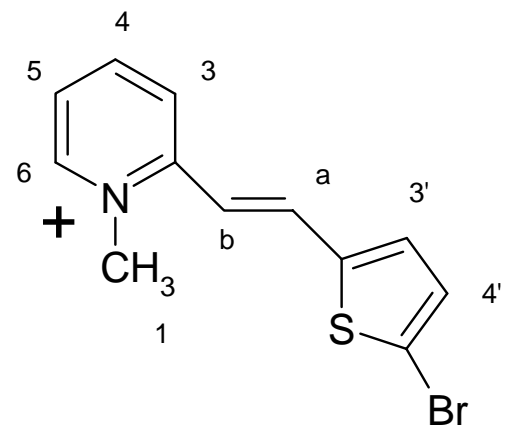

1-(4-Bromothiophen 2-yl)-2-(1-methylpyridinium 2-yl) ethylene iodide (10). 5\% $\mathrm{NaOH}$, EtOH, 10 min, reflux. Yield 84\%; (Yellow needles from aqueous-ethanol); mp 262-265 ${ }^{\circ} \mathrm{C} ;{ }^{1} \mathrm{H}$ NMR (DMSO-d D $_{6}, \delta=8.03$ (d, $\left.J=16 \mathrm{~Hz}, 1 \mathrm{H}, \mathrm{a}-\mathrm{H}\right), 7.39$ (d, J=16 Hz, 1H, b-H), 4.33 (s, N Me), 8.43 (d, $J=7.5 \mathrm{~Hz}, 1 \mathrm{H}, 3-\mathrm{H}), 8.47$ (t, $J=8 \mathrm{~Hz}, 1 \mathrm{H}, 4-\mathrm{H}), 7.88$ (t, $J=6 \mathrm{~Hz}, 1 \mathrm{H}, 5-\mathrm{H}), 8.89$ (d, $J=6 \mathrm{~Hz}$, $1 \mathrm{H}, 6-\mathrm{H}), 7.94$ (s, 1H, 3'-H), 7.77 (s, 1H, 5'-H). MS: ESI $\left(\mathrm{M}^{+}=281\right)$. Anal. Calcd for $\mathrm{C}_{12} \mathrm{H}_{11}$ BrINS: C, 35.32; H, 2.72; N, 3.43; S, 7.86. Found: C, 35.26; H, 2.78; N, 3.46; S, 7.80. 


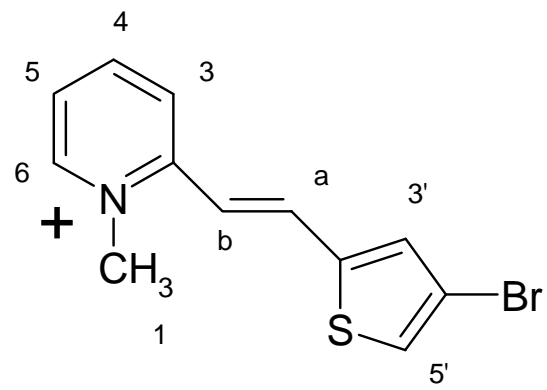

1-(1-Methylpyrrol -2-yl)- 2-(1-methyl-pyridinium-2-yl) ethylene iodide (11). 10\% $\mathrm{NaOH}$, $\mathrm{EtOH}, 20$ min, reflux. Yield 39\%; (Red needles from ethanol); mp 226-228 ${ }^{\circ} \mathrm{C}$ (lit., ${ }^{5} 187-188^{\circ} \mathrm{C}$ as $\mathrm{CF}_{3} \mathrm{SO}_{3}{ }^{-}$); ${ }^{1} \mathrm{H}$ NMR (DMSO- $\mathrm{d}_{6}$, NOE on irradiation of $\mathrm{N}^{+} \mathrm{Me}$ provided unambiguous assignment for b-H and 6-H) $\delta=7,88(\mathrm{~d}, J=15.5 \mathrm{~Hz}, 1 \mathrm{H}, \mathrm{a}-\mathrm{H}), 7,11(\mathrm{~d}, J=15.5 \mathrm{~Hz}, 1 \mathrm{H}, \mathrm{b}-\mathrm{H})$, 4.25 (s, 3H ,1-H), 8.55 (d, J=9 Hz, 1H, 3-H),8.33 (t, 1H, 4-H), 7,69 (t, 1H, 5-H), 8.72 (d, J=6.5 Hz, 1H, 6-H), 7.08 (m, 2H, 3'-H, 5'-H), 6.23 (m, 1H, 4'-H), 3.81 (s, 3H, N-Me). MS : positive FAB $\left(\mathrm{M}^{+}=198.8\right)$. Anal. Calcd for $\mathrm{C}_{13} \mathrm{H}_{15} \mathrm{IN}_{2}$ : C, 47.87; H, 4.63; N, 8.59. Found: C, 47.95; H, 4.60; $\mathrm{N}, 8.71$.

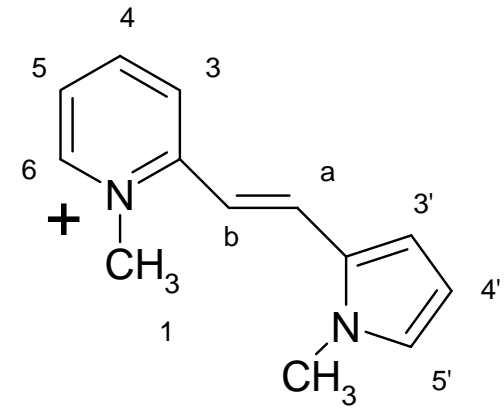

Formation of 1-(Tiazol-2-yl)-2-(1-methylpyridinium-2-yl) ethylene iodide (12) and of biscation (13). 1,2 Dimethylpyridinium iodide $\left(6.4 \times 10^{-4} \mathrm{~mol}\right)$ were dissolved in $2 \mathrm{~mL}$ hot ethanol. On addition of a drop $5 \% \mathrm{NaOH}$ and an equimolar amount of 2-tiazolcarboxaldehyde a white precipitate was noticed. After filtration and recrystallization from ethanol, $0.06 \mathrm{~g}$. yellow needles, were obtained. The solid was a mixture of the expected monocation 12 and of biscation 13 (1:5 ratio), as evidenced by ${ }^{1} \mathrm{H}$ NMR. By subtraction of the ${ }^{1} \mathrm{H}$ NMR spectrum of pure biscation 13 (see below), it is possible to assign the follows signals to monocation $12 .{ }^{1} \mathrm{H}$ NMR (DMSO- $\mathrm{d}_{6}$ ) , (compound 12): $\delta=8.13(\mathrm{~d}, J=15.5 \mathrm{~Hz}, 1 \mathrm{H}, \mathrm{a}-\mathrm{H}), 7.78$ (d, $\left.J=17 \mathrm{~Hz}, 1 \mathrm{H}, \mathrm{b}-\mathrm{H}\right), 4.33$ (s, 3H, N ${ }^{+} \mathrm{Me}$ ), 8.58 (d, J=1 Hz,1H, 3-H), 8.48 (t, 1H, 4-H), 7,69 (m, 3H, 5-H, 4'-H, 5'-H), 8.98 (d, $J=4 \mathrm{~Hz}, 1 \mathrm{H}, 6-\mathrm{H})$. MS: ESI, FAB $\left[\mathrm{M}^{+}(\mathbf{1 2})=203, \mathrm{M}^{2+}(\mathbf{1 3})=301.1\right]$.

Biscation 13 was then synthesized according to the following procedure: 1,2 Dimethylpyridinium iodide $\left(6.6 \times 10^{-4} \mathrm{~mol}\right)$ were dissolved in $2 \mathrm{~mL}$ refluxing ethanol. One drop $10 \% \mathrm{NaOH}$ was added with stirring and after few minutes $0.03 \mathrm{~mL}$ 2-thiazolcarboxaldehyde 
(3.28 $\left.\times 10^{-4} \mathrm{~mol}\right)$ were added. The resulting white precipitate was filtered and identified as pure biscation 13. Yield 49\%; (Yellow needles from ethanol); mp $>200{ }^{\circ} \mathrm{C} ;{ }^{1} \mathrm{H}$ NMR (DMSO-d $\mathrm{d}_{6}$ ), $\delta=$ 7.60 (d, $J=3,3 \mathrm{~Hz}, 1 \mathrm{H}, 5^{\prime}-\mathrm{H}$ ), 7.76 (d, $J=3.3 \mathrm{~Hz}, 1 \mathrm{H}, 4^{\prime}-\mathrm{H}$ ), 4.56 (m, 1H, a-H), 3.80 (t, $J=1.2 \mathrm{~Hz}$, 4H, b-H), 4.34 (s, 6H, N ${ }^{+} \mathrm{Me}$ ), 9.00 (d, $\left.J=6 \mathrm{~Hz}, 2 \mathrm{H}, 6-\mathrm{H}\right), 7.98$ (t, $\left.J=1 \mathrm{~Hz}, 2 \mathrm{H}, 5-\mathrm{H}\right), 8.45$ (t, $J=7$ $\mathrm{Hz}, 2 \mathrm{H}, 4-\mathrm{H}), 7.93$ (d, $J=8 \mathrm{~Hz}, 2 \mathrm{H}, 3-\mathrm{H})$, MS: positive FAB $\left(\mathrm{M}^{2+}=301.1\right)$. Anal. Calcd for $\mathrm{C}_{18} \mathrm{H}_{21} \mathrm{I}_{2} \mathrm{~N}_{3} \mathrm{~S}$ : C, 38.25; H, 3.74; N, 7.43; S, 5.67. Found: C, 38.33; H, 3.82; N, 7.40; S, 5.68.

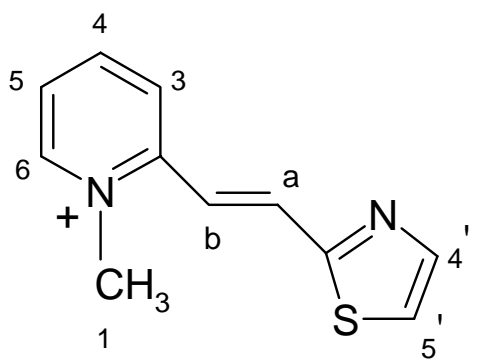

12

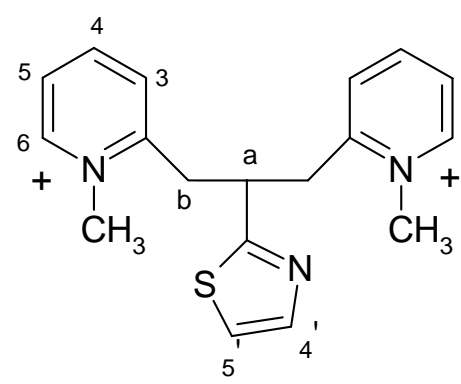

13

\section{Biological assays}

Human cell lines (LNCap and MCF7). Human prostate adenocarcinoma cells (LNCap) were grown in RPMI 1640. Human mammary adenocarcinoma (MCF7) were grown in Dulbecco's MEM (DMEM), 1.0 g/l D-glucose. Each medium was supplemented with 10\% (vol/vol) heatinactivated fetal bovine serum, $2 \mathrm{mM}$ L-Alanyl-L-Glutamine, penicillin-streptomycin (50 units$50 \mu \mathrm{g}$ for $\mathrm{ml}$ ) and incubated at $37{ }^{\circ} \mathrm{C}$ in humidified atmosphere of $5 \% \mathrm{CO}_{2}, 95 \%$ air. The culture medium was changed twice a week.

Treatment with antitumor agents and MTT colorimetric assay. Each human cancer cell line (5x103 cells $/ 0.33 \mathrm{~cm} 2)$ were plated in 96 well plates "Nunclon TM Microwell TM" (Nunc) and were incubated at $37^{\circ} \mathrm{C}$. After $24 \mathrm{~h}$, cells were treated with the compounds 1, 3, 4, 5, 6 and 11 (final concentration 0.01-100 $\mu \mathrm{M}$ ). Untreated cells were used as controls. Microplates were incubated at $37{ }^{\circ} \mathrm{C}$ in humidified atmosphere of $5 \% \mathrm{CO}_{2}, 95 \%$ air for 3 days and then cytotoxicity was measured with colorimetric assay based on the use of tetrazolium salt MTT (3-(4,5-dimethylthiazol-2-yl)-2,5-diphenyl tetrazolium bromide). ${ }^{12}$ The results were read on a multiwell scanning spectrophotometer (Multiscan reader), using a wavelength of $570 \mathrm{~nm}$. Each value was the average of 8 wells (standard deviations were less than 20\%). The $\mathrm{GI}_{50}$ value was calculated according to NCI: thus, $\mathrm{GI}_{50}$ is the concentration of test compound where $100 \mathrm{x}(\mathrm{T}$ $\mathrm{T} 0) /(\mathrm{C}-\mathrm{T} 0)=50$ ( $\mathrm{T}$ is the optical density of the test well after a 48-h period of exposure to test drug; $\mathrm{T} 0$ is the optical density at time zero; $\mathrm{C}$ is the control optical density). 


\section{Acknowledgements}

Financial support of the University of Catania, and of MIUR is gratefully acknowledged.

\section{References}

1. Ballistreri, A.; Gregoli, L.; Musumarra, G.; Spalletti, A. Tetrahedron 1998, 54, 9721.

2. Fichera, M.; Fortuna, C. G.; Impallomeni, G.; Musumarra, G. Eur. J. Org. Chem. 2002, 145.

3. Barresi, V.; Condorelli, D. F.; Fortuna, C. G.; Musumarra, G.; Scirè, S. Bioorg. Med. Chem. 2002, 10, 2899.

4. Dulcic, A.; Flytzanis, C.; Tang, D. Pepin, C. L.; Fetizon, M.; Hoppilliard, Y. J. Chem. Phys. 1981, 74, 1559.

5. Bradamante, S.; Facchetti, A.; Pagani, G. A. J. Phys. Org. Chem. 1997, 10, 514.

6. Abbotto, A.; Beverina, L.; Bozio, R.; Bradamante, S.; Ferrante, C.; Pagani, G.A.; Signorini, R. Adv. Mater. 2000, 12, 1963.

7. Bruce, G. T.; Cooksey, A. R.; Morgan, K. J. Chem. Soc. Perkin II 1975, 551.

8. Noyce, D. S.; Fike, S. A. J. Org. Chem. 1973, 38, 3316.

9. Fichera, M.; Gregoli, L.; Musumarra, G. J. Phys. Org. Chem. 2000, 13, 344.

10. Koelsch, C. F. J. Am. Chem. Soc. 1944, 66, 2126.

11. Acheson, R. M.; Harrison, D. R. J. Chem. Soc., C 1970, 1764.

12. Mosmann, T. J. J. Immunol. Meth. 1983, 65, 55. 